\title{
Performance of Response Based One Shot Controls Handling Missing Phasor Measurements
}

\author{
Niraj Dahal, Student Member, IEEE \\ Steven M. Rovnyak, Member, IEEE \\ Department of Electrical and Computer Engineering \\ Purdue School of Engineering and Technology, IUPUI \\ Indianapolis, Indiana 46202
}

\begin{abstract}
With the advent of real-time PMU data acquisition technology, the possibility of solutions to several instability problems in power system has increased. However, PMUs may undergo different data quality issues like recording bad data or missing data. Some paper mentions about 5-10\% of missing samples in some historical PMU's dataset. This paper assumes $0-10 \%$ of missing phasor samples by randomly deleting measurements and explores imputation methods of handling missing data in real time. The simulation is carried out in a DT-based stability prediction and one-shot control scheme of WECC's 176-bus model. Several control performances are evaluated to decide a useful method of missing data recovery for the response based one shot control scheme. A PMU data quality issue is not limited to missing samples only but also interference with noises. Later part of this paper performs simulation considering noisy phasor measurements. A $45 \mathrm{~dB}$ of Gaussian distributed noise is deliberately added to phasor samples and simulation is performed with different DT indices and thresholds for real time stability prediction and control actuation.
\end{abstract}

Index Terms-Decision trees, one shot control, phasor measurement units, power system transient stability, response based system, wide area control

\section{INTRODUCTION}

A wide area monitoring system (WAMS) technology involves several synchronized phasor measurement units (PMUs) across the network that measure and collect phasor samples to a central location. These samples serve important role in monitoring and analyzing different characteristics of a power system such as state estimation, fault detection, etc. The concept of synchronized phasor measurements and their application to improve monitoring, protection and control of a power system is well explained in [1]. The advent of realtime PMU data acquisition has provided solutions to various instability problems, however, one cannot ignore several data quality issues associated with this technology. Data quality issues, for example, recording non-numeric data, bad data or simply missing data might occur due to problems related to synchronization signals, equipment errors and malfunction, communication noises, etc [2]. Reference [3] mentions that on an average, $5-10 \%$ of missing samples in some historical PMUs data sets have been recorded.

Monitoring and analysis of phasor samples with missing values has significant effect on the overall quality of the result

This material is based upon the work supported by the National Science Foundation under Award ECCS-1711521 and therefore should be handled properly. For a real time power system control, general methods of missing data handling like deletion technique, mean value method or maximum frequency method are not likely to generate appropriate results because they do not involve extrapolation of previous samples. Methods that use extrapolation technique like zero order hold $(\mathrm{ZOH})$, first order hold $(\mathrm{FOH})$ and Lagrange extrapolation explained in [2], [4], [5], [6] could possibly be useful to recover missing samples from PMUs.

Lately security assessment with missing phasor measurements has been widely carried out using data mining algorithms like ensemble decision trees (EDT) with bagging, boosting, surrogate split, etc. [7], [8], [9], [10]. The surrogatesplit algorithm uses several alternate predictors with higher association to the primary classifier to create a split in case when primary predictor is missing. The methods of bagging and boosting rather work more accurately with several full grown DTs and mostly consume large memory space. Another limitation of EDT is that it restricts the users to retrieve the complete data-set and therefore gives lower flexibility to try out different other methods for analysis of phasor samples. The advantage of simulation-based imputation methods like $\mathrm{ZOH}$, $\mathrm{FOH}$, or Lagrange polynomial method is that the imputation phase and the analysis phase can be totally separated allowing to work on a complete data-set [11]. The recovered data-set can then be used for creating small DTs with one or two nodes using limited predictors and low memory space.

The scope of this paper does not include training ensemble decision trees using missing predictors, but uses imputation techniques to recover missing values from $0-10 \%$ directly on the test sets. The recovered phasor measurements and other indices [12], [13] are fed to response based DTs [14] to trigger one shot stabilizing control [15]. Preliminary methods described in this paper first train predictors to create single node DTs for instability control without event detection. Later methods train another single node DT with an ability to detect end of event that is augmented to the control DT to perform control with event detection [13], [16]. The main purpose of augmenting event detection DT with the control DT is to create a less 'trigger happy' DT that actuates control for a limited number of severe cases only, thereby reducing the number of unnecessary controls. The notion of 'trigger happy' DTs is explained in [13]. 


\section{Proposed Missing Data Imputation Techniques}

Data hold methods are simple extrapolation techniques that can be used for recovering missing PMU's samples. A zero order hold $(\mathrm{ZOH})$ technique holds a previous sample in the missing place until the arrival of next sample. A first order hold $(\mathrm{FOH})$ method recovers the missing sample by linear approximation of two recent available samples. Since we are assuming a temporal correlation of phasor samples from a particular channel, we also use Lagrange extrapolation technique to recover missing PMU's measurements [17].

\section{A. Data Holds}

Holding a data generates a continuous time signal $g(t)$ from a discrete-time sequence $x(k T)$ [5]. Let us consider a signal $g(t)$ during a time interval $t$ such that $k T \leq t \leq(k+1) T$, then $g(t)$ can be approximated by a polynomial as-

$$
\begin{array}{r}
g(k T+\tau)=a_{0}+a_{1} \tau+\ldots+a_{n-1} \tau^{n-1}+a_{n} \tau^{n} \\
g(k T+\tau)=x(k T)+a_{1} \tau+\ldots+a_{n-1} \tau^{n-1}+a_{n} \tau^{n}
\end{array}
$$

where $0 \leq \tau \leq T$.

It can be seen that at $\tau=0, g(k T)=x(k T)$. An $n^{t h}$ order hold circuit uses $(n+1)$ discrete data to generate $g(k T+\tau)$.

1) Zero Order Hold ( $\mathrm{ZOH})$ : $\mathrm{ZOH}$ performs signal reconstruction by holding a recent value until next sample arrives [5]. In (1), if $n=0$, a zero-order hold is obtained such that

$$
g(k T+\tau)=x(k T)
$$

where $0 \leq \tau \leq T$ and $k=0,1,2, \ldots$

2) First Order Hold (FOH): FOH performs signal reconstruction by using $(n+1)=2$ recent discrete data [5]. Therefore, in (1) for $\mathrm{n}=1$,

$$
\begin{gathered}
g(k T+\tau)=x(k T)+a_{1} \tau \\
g(k T+\tau)=x(k T)+\frac{x(k T)-x((k-1) T)}{T} \tau
\end{gathered}
$$

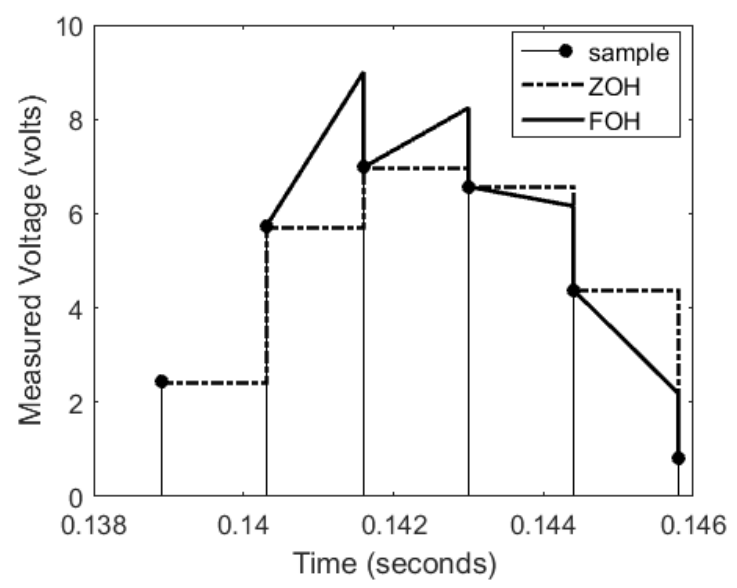

Fig. 1. Data hold methods

Fig. 1 shows an example for measured data samples and their corresponding $\mathrm{ZOH}$ and $\mathrm{FOH}$ signals.

\section{B. Lagrange Polynomial Method}

Lagrange polynomial method gives the lowest degree polynomial passing through given set of points $\left(x_{j}, y_{j}\right)$ where each $x_{j}$ is different than the other such that $1 \leq j \leq(n+1)$.

Lagrange interpolating polynomial is described in [2][6][18] and can be represented as follows:

$$
L(x)=\sum_{j=1}^{n} y_{j} l_{j}(x)=\sum_{j=1}^{n} y_{j} \prod_{k=1, k \neq j}^{n} \frac{x-x_{k}}{x_{j}-x_{k}}
$$

where $x_{j} \neq x_{k}$ and $l_{j}(x)$ is the coefficient in the Lagrange polynomial.

Considering $\mathrm{n}=3$, the Lagrange polynomial can be expanded as follows:

$$
\begin{aligned}
L(x)=y_{1} \frac{\left(x-x_{2}\right)\left(x-x_{3}\right)}{\left(x_{1}-x_{2}\right)\left(x_{1}-x_{3}\right)}+y_{2} \frac{\left(x-x_{1}\right)\left(x-x_{3}\right)}{\left(x_{2}-x_{1}\right)\left(x_{2}-x_{3}\right)} \\
+y_{3} \frac{\left(x-x_{1}\right)\left(x-x_{2}\right)}{\left(x_{3}-x_{1}\right)\left(x_{3}-x_{2}\right)}
\end{aligned}
$$

If $\mathrm{P} 1, \mathrm{P} 2, \mathrm{P} 3$ denotes three recent preceding sample positions, then a missing point can be recovered by strict extrapolation (SE-lag) of data points from these positions.

TABLE I

STRICT LAGRANGE EXTRAPOLATION (SE-LAG)

\begin{tabular}{c|c|c|c|} 
P3 & P2 & P1 & Missing data \\
\hline$x_{k-3}$ & $x_{k-2}$ & $x_{k-1}$ & $x_{k}$ \\
$y_{k-3}$ & $y_{k-2}$ & $y_{k-1}$ & $y_{k}$
\end{tabular}

The $x_{k}$ 's are time domain samples at $30 \mathrm{~Hz}$ and the $y_{k}$ 's are phasor measurements corresponding to $k^{t h}$ sample.

\section{CAse Study}

The proposed methods for missing data recovery is experimented in a 29-machine, 176 bus model of Western Electricity Coordinating Council (WECC). PMUs are installed in 17 buses. For data obtained from 385 six-seconds of simulations, decision trees are trained to detect end of events and generate one shot control criteria. This training set is same as the one used in [12]. The one shot control combination used in this research is same as in [13] which is $500 \mathrm{MW}$ of generator tripping on two AC buses and corresponding $500 \mathrm{MW}$ of load shedding on other two buses.

\section{A. Training the Control DT}

Reference [13] deduces that use of bus angle velocities yields better success rates in predicting if an event needs control or no control. Therefore, the input vectors used for training the control DT in our case is bus angle velocities of 17 PMU buses obtained from 385 six-second simulations of the model. A binary target is used to classify each time sample of the events as 'CONTROL' for unstable events and 'NO CONTROL' for stable events. An unstable event is the one whose maximum generator angle difference is greater than a certain threshold value like 300 degrees explained in [19], otherwise we classify them as stable. 
This paper uses a rule-set derived in [13] that if the bus angle velocity of $9^{\text {th }}$ bus at any time sample is greater than or equal to 80 i.e. $\left(\theta_{\operatorname{dot} 9}>=80\right)$. the event is set to 'CONTROL'.

\section{B. Training the Event Detection DT}

Whenever a severe short circuit fault occurs in a transmission system, some of the bus voltage magnitudes reduce below 1 per unit (pu) as shown in Fig. 2. This gives rise to large variance of bus voltage magnitudes. As soon as the faulty line is removed, the voltages tend to return to $1 \mathrm{pu}$ and hence the variance decreases. The derivative of bus voltage magnitude variance (bmvardot) thus has a large positive spike during the fault and large negative spike right after the fault is cleared which makes it a good indicator for short circuit faults. The nature of bmvardot can be seen in Fig. 8. The variance here is infact standard deviation. The mathematics of bmvardot index is explained in [13].

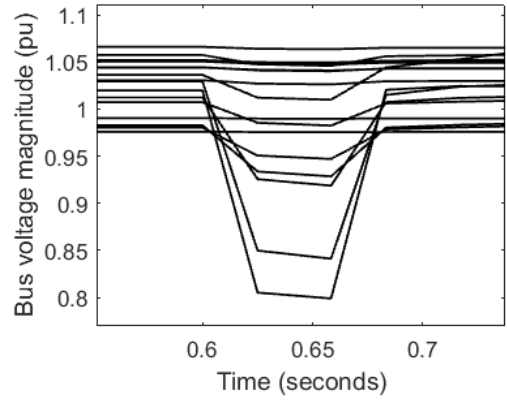

Fig. 2. Bus voltage magnitudes during a fault

Reference [13] experiments that the DT trained using bmvardot better detect end of faults when its value is less than or equal to a threshold of -0.03 pu (bmvardot $<=-0.03$ ). This event detection DT is combined to one of the control DTs to actuate control with event detection as shown in the Fig. 3. The timer to actuate control is set to maximum of 5 samples i.e. 0.167 seconds after detection of an event.

\section{Test Results And Analysis}

All missing data handling techniques are tested in a set of 480 single line to ground (SLG) fault test contingencies [13] for six seconds simulation in TSAT (Transient Security Assessment Tool). Pseudo-random number generators like $\operatorname{rand}()$ function in MATLAB is used to randomly delete samples of bus voltage magnitudes and bus voltage angles from $0-10 \%$. With the recovered phasor measurements, simulations are then performed to actuate one shot control with or without event detection. When the control DT is satisfied, a 100 milliseconds actuation delay is applied before triggering the one shot control combination.

\section{A. Test Results without Event Detection}

Table II shows the number of events controlled by each imputation method subjected to $0-10 \%$ of missing phasor measurements. It can be observed that the number of events controlled for ZOH and SE-Lag is increasing gradually while

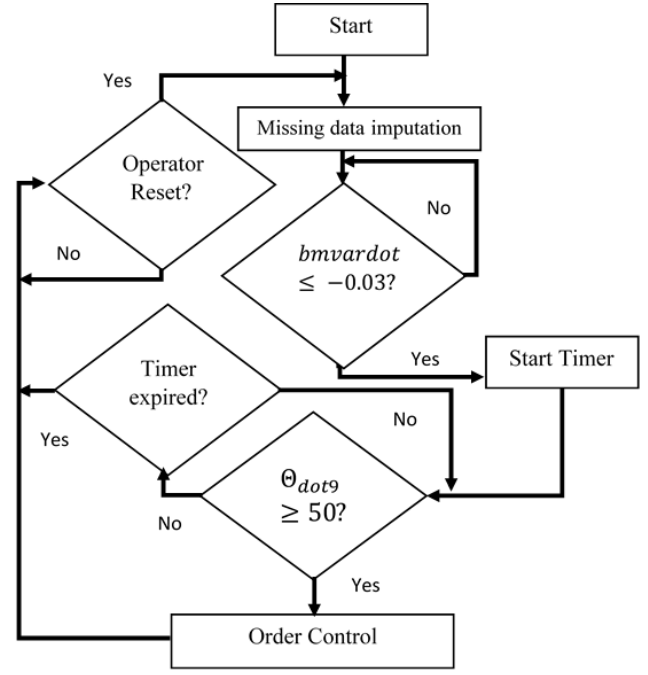

Fig. 3. Control methodology with event detection

for FOH, it remains lesser and almost constant. The success rate of any method is higher only if, for the same number of events controlled, the method stabilizes more number of events. Fig. 4 shows the comparison of success rates. The $\mathrm{FOH}$ method has almost the same success rate for higher percentage of data missed, while for other methods, the success rate decreases gradually.

TABLE II

EVENTS CONTROLLED WITHOUT EVENT DETECTION

\begin{tabular}{|c|c|c|c|c|c|c|c|c|c|c|c|}
\hline$\%$ Miss & 0 & 1 & 2 & 3 & 4 & 5 & 6 & 7 & 8 & 9 & 10 \\
\hline ZOH & 195 & 212 & 224 & 230 & 240 & 249 & 253 & 260 & 264 & 269 & 270 \\
\hline FOH & 195 & 195 & 194 & 194 & 194 & 195 & 195 & 195 & 195 & 197 & 197 \\
\hline SE-Lag & 195 & 335 & 388 & 407 & 423 & 436 & 448 & 449 & 461 & 468 & 471 \\
\hline
\end{tabular}

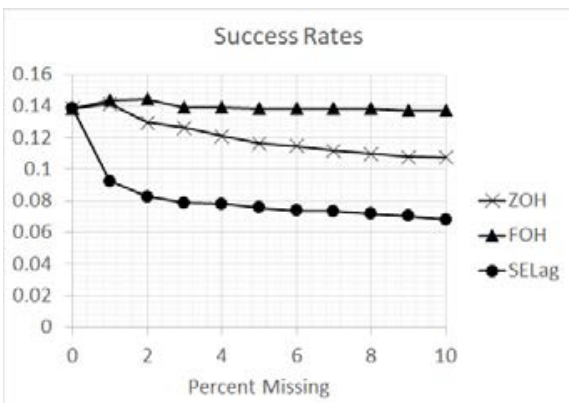

Fig. 4. Success rates without event detection

The reason that $\mathrm{ZOH}$ method did not give better success rate than $\mathrm{FOH}$ is because of its nature of holding the exact previous measurement in the missing place until the arrival of next sample. For ZOH, the difference between two samples at the point of recovery becomes zero followed by a difference almost twice as large as it should be. This large derivative value causes the DT to actuate control for more number of events. This can be illustrated from Fig. 5. We consider a missing sample from 9th PMU bus at around 0.78 seconds 
and recover it using $\mathrm{ZOH}$ and $\mathrm{FOH}$. Due to previous sample held by $\mathrm{ZOH}$, the value of bus angle velocity at the recovery point is very low around $4.5 \mathrm{deg} / \mathrm{s}$. There is a spike in the velocity right after the recovery point up to $52 \mathrm{deg} / \mathrm{s}$ thereby crossing the threshold of $\theta_{\operatorname{dot} 9}>50$ and actuating control at around 0.82 seconds. This situation occurs frequently with increase in percentage missing and worsens when consecutive samples are missing in the data-set. The behavior of $\mathrm{FOH}$ tends to reduce this error.

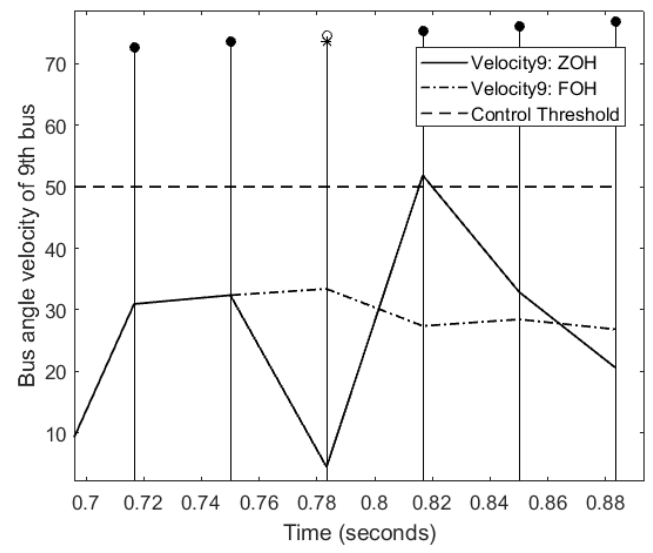

Fig. 5. ZOH behavior to trigger more controls

The fact of controlling a large number of events allows $\mathrm{ZOH}$ to stabilize few additional events but trigger substantially higher unnecessary controls. Unnecessary controls are those simulations where control is actuated to a self-recovering event and are not desired. Simulation result shows even larger unnecessary controls for SE-Lag method as shown in Fig. 6.

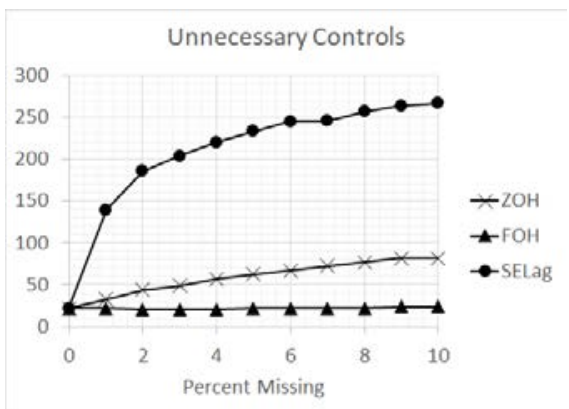

Fig. 6. Unnecessary controls without event detection

\section{B. Test Results with Event Detection}

Table III shows the number of events controlled with event detection for different percent of data missed. The result is similar to Table II but with lower number of events controlled. This is expected because with the augmentation of event detection DT, the control DT is checked within a specified timer only. This modification also results in lower number of unnecessary controls and higher success rates. Table IV shows the number of unnecessary controls for $0-10 \%$ of data missed which is substantially lower than the results obtained from control without event detection.

Fig. 7 shows the success rates for different methods.

TABLE III

EVENTS CONTROLLED WITH EVENT DETECTION

\begin{tabular}{|c|c|c|c|c|c|c|c|c|c|c|c|}
\hline$\%$ Miss & 0 & 1 & 2 & 3 & 4 & 5 & 6 & 7 & 8 & 9 & 10 \\
\hline ZOH & 96 & 97 & 98 & 100 & 102 & 103 & 104 & 105 & 105 & 106 & 108 \\
\hline FOH & 96 & 98 & 99 & 98 & 98 & 98 & 97 & 97 & 98 & 102 & 103 \\
\hline SE-Lag & 96 & 104 & 113 & 124 & 133 & 143 & 152 & 158 & 163 & 173 & 184 \\
\hline
\end{tabular}

TABLE IV

UNNECESSARY CONTROLS WITH EVENT DETECTION

\begin{tabular}{|c|c|c|c|c|c|c|c|c|c|c|c|}
\hline$\%$ Miss & 0 & 1 & 2 & 3 & 4 & 5 & 6 & 7 & 8 & 9 & 10 \\
\hline ZOH & 6 & 6 & 7 & 9 & 10 & 11 & 12 & 12 & 13 & 14 & 15 \\
\hline FOH & 6 & 7 & 7 & 6 & 6 & 6 & 5 & 5 & 5 & 7 & 8 \\
\hline SE-Lag & 6 & 13 & 20 & 29 & 37 & 47 & 55 & 59 & 61 & 68 & 78 \\
\hline
\end{tabular}

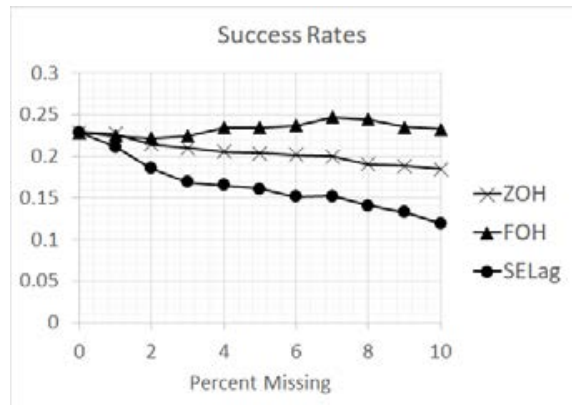

Fig. 7. Success rates with event detection

\section{Test Results with Addition of Noise}

The probability distribution of PMU noise and its quantification has been studied in [20] which generalizes that Gaussian distributed noise with Signal-Noise ratio (SNR) of $45 \mathrm{~dB}$ is realistic for simulating purposes. We perform addition of white Gaussian noise with zero mean and unity variance to our phasor samples like in [16, p. 38] and find new thresholds for event detection and control rule.

After addition of noise, the calculated indices will also contain noise as shown in the Fig. 8. The initial rule for event detection such as bmvardot $<=-0.03$ causes the DT to detect event way earlier in the simulation than the actual occurrence of fault. Adjusting the threshold value to more negative values like less than -0.10 or -0.12 eliminates the chance of early event detection as shown in Fig. 9 and improves the control performance. Other indices like derivative of average of bus voltage magnitudes (bmavgdot) [13] plotted in Fig. 8 can also be used for event detection as it filters out the noise and proves equally useful as bmvardot.

Different values for event detection threshold were adjusted with control rule of $\theta_{\text {dot } 9}>=80$ to find performance for 480 SLG test faults as in Table V. It shows results for number of events stabilized, events controlled, unnecessary controls and success rate respectively. 


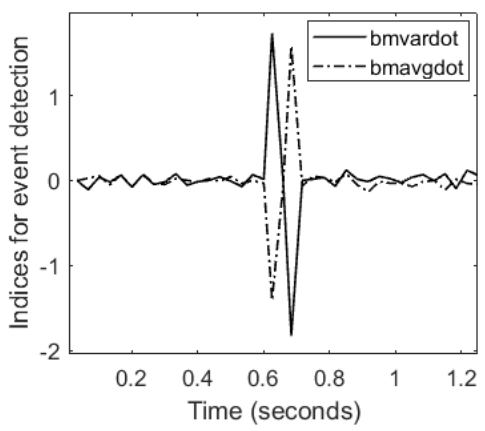

Fig. 8. Indices for event detection with noise

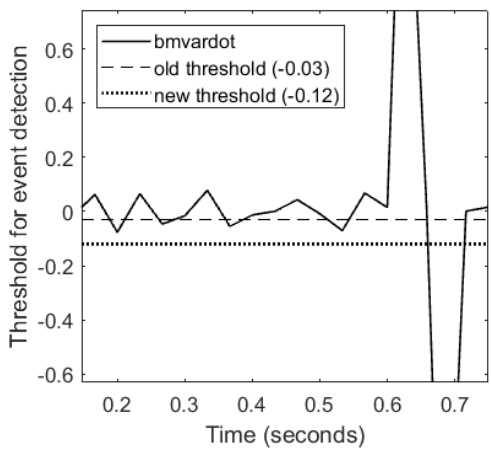

Fig. 9. Adjusting new threshold for bmvardot

\section{CONCLUSION}

Previous work done for missing data handling in [17][21] mentions about coding error due to which the $\mathrm{ZOH}$ results are expected to be more accurate for smaller percent missing only. Similarly, the work done in [17] mentions about an unrealistic approach of using future values for real time missing data recovery. The work done in this paper corrects these mistakes and deduces that First Order Hold (FOH) is more useful in recovering missing values for a response based scheme as in [12], [13], [14]. The success rate with $10 \%$ missing data for FOH method is almost $23 \%$ while for $\mathrm{ZOH}$, it is $18 \%$ only. The reason that $\mathrm{ZOH}$ method does not prove better than $\mathrm{FOH}$ is because it contributes to sufficiently higher unnecessary controls and lower success rate. This paper in addition performs simulation with noisy phasor measurements which show that the control combination used here is robust enough to handle at least $45 \mathrm{~dB}$ of Gaussian noise in PMUs' measurements. The performance shows a success rate of around $16-18 \%$. A future enhancement of this work could be using data mining algorithms like ensemble DTs with missing predictors like in [7], [8], [9], [10] and comparing the results with imputation methods described in this paper.

\section{REFERENCES}

[1] J. D. L. Ree, V. Centeno, J. Thorp, and A. Phadke, "Synchronized phasor measurement applications in power systems," IEEE Transactions on Smart Grid, vol. 1, no. 1, pp. 20-27, June 2010.

[2] C. Hunag, F. Li, and e. L. Zhan, "Data quality issues for synchrophasor applications part ii: Problem formulation and potential solutions," $J$. Mod. Power System Clean Energy, 2016.
TABLE V

CONTROL PERFORMANCE WITH NOISE

\begin{tabular}{|c|c|c|c|c|}
\hline Event det DT & Nstab & Ncntrol & Nunne & Success \\
\hline bmvardot $<-0.20$ & 19 & 116 & 22 & 0.164 \\
bmvardot $<-0.16$ & 19 & 115 & 22 & 0.165 \\
bmvardot $<-0.12$ & 19 & 115 & 27 & 0.165 \\
\hline bmavgdot $>0.05$ & 5 & 32 & 13 & 0.156 \\
bmavgdot $>0.07$ & 11 & 60 & 23 & 0.183 \\
bmavgdot $>0.15$ & 19 & 114 & 22 & 0.167 \\
\hline
\end{tabular}

[3] M. Wang, "Data quality management of synchrophasor data in power system by exploiting low-dimensional models," 51st Annual Conference on Information Sciences and Systems, Baltimore, MD, pp. 1-2, 2017.

[4] Z. Zhang and K. T. Chong, "Comparison between first-order hold with zero order hold in discretization of input-delay nonlinear systems," International Conference on Control, Automation and Systems, Seoul, pp. 2892-2896, 2007.

[5] Data hold methods. Chapter3.pdf. [Online]. Available: http://web.cecs. pdx.edu/ tymerski/ece452/Chapter3.pdf

[6] P. Manembu, A. Kewo, and B. Welang, "Missing data solution of electricity consumption based on langrange interpolation case study:intelligensia data monitoring," 2015 International Conference on Electrical Engineering and Informatics (ICEEI), Denpasar, pp. 511516,2015

[7] M. He, V. Vittal, and J. Zhang, "Online dynamic security assessment with missing pmu measurements: A data mining approach," IEEE Transactions on Power Systems, vol. 28, pp. 1969-1977, May 2013.

[8] S. Samantaray, I. Kamwa, and G. Joos, "Ensemble decision trees for phasor measurement unit-based wide-area security assessment in the operations time frame," IET Generation, Transmission and Distribution, vol. 4, no. 12, pp. 1334-1348, June 2010.

[9] A. C. Adewole and R. Tzoneva, "Surrogate-splits ensembles for realtime voltage stability assessment in the presence of missing synchrophasor measurements," IET Science, Measurement and Technology, vol. 11, no. 5, pp. 545-552, May 2017.

[10] C. Liu, C. Bak, Z. Chen, and P. Lund, "Dynamic security assessment of western danish power system based on ensemble decision trees," 12th IET International Conference on Developments in Power System Protection (DPSP 2014), pp. 1-6, 2014.

[11] A. Feelders, Handling Missing Data in Trees: Surrogate Splits or Statistical Imputation? Springer,Berlin, Heidelberg, 1999.

[12] Q. Gao and S. M. Rovnyak, "Decision trees using synchronized phasor measurements for wide-area response based control," IEEE Transactions on Power System, vol. 26, May 2011.

[13] S. Rovnyak, D. W. Longbottom, D. C. Vasquez, and M. N. Nilchi, Monitoring and Control using Synchrophasors in Power Systems with Renewables. Chapter 8: IET, 2017, ch. Response Based Event Detection for One Shot Wide-area Stability Controls, in press.

[14] K. Mei and S. M. Rovnyak, "Response based decision trees to trigger one shot stabilizing control," IEEE Transactions on Power System, vol. 19, pp. 531-537, February 2004.

[15] S. Rovnyak, G. Li, and K. Mei, "One-shot controls for preventing loss of synchronism," 2003 IEEE Power Engineering Society General Meeting (IEEE Cat. No.03CH37491),Toronto, Ont., vol. 4, pp. 2045-2052, 2003.

[16] D. Longbottom, "Polynomial curve fitting indices for dynamic event detection in wide-area measurement systems," MSECE Thesis, Purdue University, Indianapolis, 2012

[17] N. Dahal and S. Rovnyak, "Methods of missing data handling in one shot response based power system control," Presented at Indiana Summit Conference 2019, Indianapolis, Indiana, in press.

[18] N. Le and W. Benjapolakul, "A data imputation model in phasor measurement units on bagged averaging of multiple linear regression," IEEE Access, vol. 6, pp. 39324-39333, 2018.

[19] M. Nilchi, "Electric utility planning methods for the design of one shot stability controls," MS Thesis, Purdue University, Indianapolis, 2012.

[20] M. Brown, M. Biswal, S. Brahma, S. Ranade, and H. cao, "Characterizing and quantifying noise in pmu data," 2016 IEEE Power and Energy Society General Meeting (PESGM), Boston, MA, pp. 1-5, 2016.

[21] N. Dahal, "Methods of handling missing data in one shot response based power system control," MSECE Thesis, Purdue University, Indianapolis, 2019. 